\title{
MAGNETIC ABSORPTION OF METAL NANOPARTICLES
}

The dependences of the magnetic absorption by spheroidal metal nanoparticles on the ratio between the particle curvature radii and the angle between the spheroid symmetry axis and the magnetic field vector of an incident electromagnetic wave are plotted and theoretically analyzed. An interesting result of the research is the growth of the energy absorbed by a spheroidal nanoparticle, as it becomes more oblate.

Ke ywords: magnetic field, optics, metal nanoparticles, averaged parameters.

\section{Introduction}

The research of optical properties of nanoparticles has a long history (see, e.g., books [1-4]). In particular, the expression for the absorption cross-section of a plane electromagnetic wave with the frequency $\omega$ by a spherical nanoparticle, whose sizes are smaller than the radiation wavelength, has been known for a long time [5]:

$K(\omega)=\frac{12 \pi \omega a^{3} \epsilon^{\prime \prime}}{c}\left\{\frac{1}{|\epsilon|^{2}}+\frac{\omega^{2} a^{2}}{90 c^{2}}\right\}$.

Here, $c$ is the light velocity; $\epsilon$ the dielectric constant, which has the following form in the Drude model [3]:

$\epsilon=\epsilon^{\prime}+i \epsilon^{\prime \prime}=1-\frac{\omega_{p}^{2}}{\omega^{2}+\nu^{2}}+i \frac{\nu}{\omega} \frac{\omega_{p}^{2}}{\omega^{2}+\nu^{2}}$,

$\nu$ is the bulk collision frequency;

$\omega_{p}=\left(4 \pi n_{0} e^{2} / m\right)^{1 / 2}$

is the plasma frequency; and $e, m$, and $n_{0}$ are the electron charge, mass, and concentration, respectively. The first term in the braces on the right-hand side of formula (1) is associated with the electric component of the wave. Therefore, this kind of absorption is called electric. The second term is associated with the magnetic wave component, and the corresponding absorption is called magnetic [5].

The most general and referenced theory of the optical properties of small particles is the Mie theory [6]. This theory was developed for spherical particles in the assumption that the vector of the electric current density inside the particle, $\mathbf{j}(\mathbf{r}, t)$, is related to

(C) P.M. TOMCHUK, V.N. STARKOV, 2018 the generating field $\mathbf{E}(\mathbf{r}, t)$ by the Ohm law

$\mathbf{j}(\mathbf{r}, t)=\sigma \mathbf{E}(\mathbf{r}, t)$

where $\sigma$ is the scalar conductivity, $\mathbf{r}$ the coordinate vector, and $t$ the time. Generally speaking, relation (4) is valid for particles, whose dimensions are much larger than the electron free path [7]. In the opposite case, relation (4) becomes non-local [8]. Moreover, in the case of asymmetric particles, the conductivity transforms from the scalar quantity into the tensor one [7].

Let a metal particle be in the field of an external electromagnetic wave

$$
\left(\begin{array}{l}
\mathbf{E} \\
\mathbf{H}
\end{array}\right)=\left(\begin{array}{l}
\mathbf{E}^{(0)} \\
\mathbf{H}^{(0)}
\end{array}\right) e^{i(\mathbf{k r}-\omega t)}
$$

where $\mathbf{E}$ and $\mathbf{H}$ are the electric and magnetic, respectively, components of the wave field; and $\mathbf{k}$ is the wave vector ( $k=2 \pi / \lambda$, where $\lambda$ is the wave length). Then the solution of the problem to find the current density vector $\mathbf{j}(\mathbf{r}, t)$ responsible for the energy absorption includes two stages. At the first stage, we should determine internal fields generated by wave (5) in the nanoparticle. At the second stage, we should determine how those internal fields change the velocity distribution function of electrons, i.e. find a correction to the equilibrium Fermi distribution induced by the internal fields.

The internal fields induced by wave (5) in the nanoparticle depend on the nanoparticle shape. Therefore, we will assume that the nanoparticle has an ellipsoidal shape. It is convenient to develop a theory for this form, because the results obtained for the

ISSN 2071-0194. Ukr. J. Phys. 2018. Vol. 63, No. 10 
ellipsoidal form can be extended to a wide range of nanoparticle forms (from discoid to rod-like ones) by changing the curvature radii of the ellipsoid.

If the wave length $\lambda$ much exceeds the nanoparticle size, then the relationship of the internal and external fields, $\mathbf{E}^{(0)}$ and $\mathbf{H}^{(0)}$, is known [5]. In particular, if a coordinate system is oriented along the principal ellipsoid axes, the electric (potential) component of the internal field looks like [5]

$\left(E_{\mathrm{in}}\right)_{j}=\frac{E_{j}^{(0)}}{1+L_{j}[\epsilon(\omega)-1]}$,

where $L_{j}$ is the $j$-th diagonal component of the depolarization tensor. Analogously, the eddy component of the electric field induced by the magnetic field $\mathbf{H}^{(0)}$ equals [7]

$\left(E_{\mathrm{ed}}\right)_{x}=i \frac{\omega}{c}\left\{\frac{z H_{y}^{(0)}}{R_{z}^{2}+R_{y}^{2}}-\frac{y H_{x}^{(0)}}{R_{x}^{2}+R_{y}^{2}}\right\}$.

The other components of the eddy field can be obtained from Eq. (7) by the cyclic permutation of the subscripts. To make sure, let us recall that $H^{(0)}=E^{(0)}$

\section{Velocity Distribution}

\section{Function of Electrons and the Energy}

\section{Absorption by Metal Nanoparticles}

The velocity distribution function of electrons in metal nanoparticles in the presence of fields (6) can be written as the sum of the equilibrium Fermi function $f_{0}(\varepsilon)$, where $\varepsilon=m v^{2} / 2$ is the electron energy, and the term $f_{1}(\mathbf{r}, \mathbf{v})$ that is determined from the solution of the kinetic equation linearized with respect to the field,

$(\nu-i \omega) f_{1}+\mathbf{v} \frac{\partial f_{1}}{\partial \mathbf{r}}+e \mathbf{F}(\mathbf{r}) \mathbf{v} \frac{\partial f_{0}}{\partial \varepsilon}=0$.

The function $f_{1}(\mathbf{r}, \mathbf{v})$ must also satisfy the boundary condition

$\left.f_{1}(\mathbf{r}, \mathbf{v})\right|_{S}=0$ at $v_{n}<0$

where $v_{n}$ is the velocity component directed normally to the surface. In Eq. (8), the vector $\mathbf{F}$ describes the total (potential and eddy) field:

$\mathbf{F}=\mathbf{E}_{\mathrm{in}}+\mathbf{E}_{\mathrm{ed}} \cdot$
The solution of the boundary-value problem (8), (9) for an ellipsoidal particle has the form

$f_{1}(\mathbf{r}, \mathbf{v})=-f_{0}^{\prime}(\varepsilon) \int_{0}^{t_{0}} d \tau e^{-\tilde{\nu}\left(t_{0}-\tau\right)} e \mathbf{F}\left(\mathbf{r}^{\prime}-\mathbf{v}^{\prime}\left(t_{0}-\tau\right)\right)$,

where $\tilde{\nu}=\nu-i \omega$, and $t_{0}$ is the characteristic of Eq. (8),

$t_{0}=\frac{1}{v^{\prime 2}}\left\{\mathbf{r}^{\prime} \mathbf{v}^{\prime}+\sqrt{\left(R^{2}-r^{\prime 2}\right) v^{\prime 2}+\left(\mathbf{r}^{\prime} \mathbf{v}^{\prime}\right)^{2}}\right\}$.

The primes in Eqs. (11) and (12) mark the vectors in a deformed coordinate system, in which the ellipsoidal shape of the particle becomes spherical [7]. The coordinates in the deformed and undeformed systems are related to each other by the relations

$x_{j}=\frac{R_{j}}{R} x_{j}^{\prime}, \quad v_{j}=\frac{R_{j}}{R} v_{j}^{\prime} \quad(j=1,2,3)$,

where $R=\left(R_{1} R_{2} R_{3}\right)^{1 / 3}$, and $R_{j}$ are the curvature radii of the ellipsoid.

Knowing the velocity distribution function of electrons, we can find the current density

$\mathbf{j}(\mathbf{r}, \omega)=\operatorname{Re}\left(\frac{m}{2 \pi \hbar}\right)^{3} \int \mathbf{v} f_{1}(\mathbf{r}, \mathbf{v}) d^{3}(v)$.

According to Eqs. (10) and (11), the current density vector (14) consists of two components: electric (induced by $\mathbf{E}_{\text {in }}$ ) and magnetic (induced by $\mathbf{E}_{\text {ed }}$ ) ones:

$\mathbf{j}(\mathbf{r}, \omega)=\mathbf{j}_{e}(\mathbf{r}, \omega)+\mathbf{j}_{m}(\mathbf{r}, \omega)$.

Explicit expressions for $\mathbf{j}_{e}$ and $\mathbf{j}_{m}$ can be obtained by substituting Eq. (11) into Eq. (14).

Accordingly, the energy absorbed by the metal nanoparticle,

$W=W_{e}+W_{m}=\frac{1}{2} \operatorname{Re} \int_{V} d \mathbf{r}\left\{\mathbf{j}_{e} \mathbf{E}_{\mathrm{in}}^{*}+\mathbf{j}_{m} \mathbf{E}_{\mathrm{ed}}^{*}\right\}$,

is also the sum of the electric and magnetic components. Dividing Eq. (16) by the energy flux incident on the nanoparticle, we obtain the absorption coefficient. This is, in brief, a procedure of obtaining the light absorption coefficient for a metal nanoparticle with regard for the influence of the nanoparticle shape on the absorption. 
As a rule, an experimenter does not deal with an ensemble of absolutely identical nanoparticles, but with a certain set of them. Therefore, there arises a problem of averaging the light absorption and scattering coefficients over the nanoparticle forms. Recall that the nanoparticle shape affects not only the frequencies, but also the number of plasma resonances. In our previous work [9], we studied the dependence of the electric absorption described by the first scalar product $\mathbf{j}_{e} \mathbf{E}_{\mathrm{in}}^{*}$ in Eq. (16) on the nanoparticle form and the nanoparticle-form distribution function. In order to analyze the dependence of optical properties of an ensemble of metal nanoparticles on their shapes, we chose a model of spheroidal nanoparticles with the same volume $V$, but with different eccentricities (i.e. the forms). This form describes the simplest deviation from the spherical symmetry and characterizes this deviation by a single parameter, the eccentricity. This circumstance strongly simplifies the averaging procedure. In this work, we consider the averaging process for the magnetic absorption associated with the second scalar product $\mathbf{j}_{m} \mathbf{E}_{\text {ed }}^{*}$ in Eq. (16).

\section{Magnetic Absorption}

In what follows, we restrict the consideration to the frequency interval

$\nu \ll \omega \ll \omega_{s} \quad(s=\perp, \|)$.

For typical metals, the frequency $\nu \sim 10^{13} \mathrm{~s}^{-1}$. The inequality $\nu \ll \omega$ allows us to neglect the bulk scattering of electrons. The inequality $\omega \ll\left(\omega_{\perp}, \omega_{\|}\right)$means that we are far from plasma resonances, and the electric absorption does not "hide" the magnetic one. The substitution of Eq. (14) into Eq. (16) for the energy of magnetic absorption by a spheroidal metal nanoparticle brings us to the expression $[10,11]$

$$
\begin{aligned}
& W_{m}=\frac{9}{128} V \frac{n e^{2}}{m c^{2}} v_{\mathrm{F}} R_{\perp} \times \\
& \times\left\{\chi_{H}\left(H_{\|}^{(0)}\right)^{2}+\eta_{H}\left(\frac{R_{\|}^{2}}{R_{\perp}^{2}+R_{\|}^{2}}\right)^{2}\left(H_{\perp}^{(0)}\right)^{2}\right\} .
\end{aligned}
$$

Here, $v_{\mathrm{F}}$ is the Fermi velocity, and $H_{\|}^{(0)}$ and $H_{\perp}^{(0)}$ are the amplitudes of the magnetic field components of the electromagnetic wave $\left(H_{\perp}=\left(H_{1}^{2}+H_{2}^{2}\right)^{1 / 2}\right)$, as well as $\left.H^{(0)}=E^{(0)}\right)$ that are parallel $(\|)$ and orthogonal $(\perp)$, respectively, to the axis of the ellipsoid of revolution. Recall that, in the case of spheroid, $R_{1}=R_{2} \equiv R_{\perp}$ and $R_{3} \equiv R_{\|}$. In Eq. (18), the magnetic components were retained, because they are easier to use, while expressing the polarization dependences of the magnetic absorption by a non-spherical nanoparticle. Finally,

$$
\begin{aligned}
\chi_{H}=\left\{\begin{array}{l}
\frac{1}{8 e_{p}^{2}}\left(1+2 e_{p}^{2}\right) \sqrt{1-e_{p}^{2}}- \\
-\frac{1}{8 e_{p}^{3}}\left(1-4 e_{p}^{2}\right) \arcsin e_{p} \text { at } \rho<1, \\
-\frac{1}{8 e_{p}^{2}}\left(1-2 e_{p}^{2}\right) \sqrt{1+e_{p}^{2}}+ \\
+\frac{1}{8 e_{p}^{3}}\left(1+4 e_{p}^{2}\right) \times \\
\times \ln \left(e_{p}+\sqrt{1+e_{p}^{2}}\right) \text { at } \rho>1
\end{array}\right. \\
\eta_{H}=\left\{\begin{array}{l}
-\frac{1}{4 e_{p}^{2}}\left(1-8 e_{p}^{2}+4 e_{p}^{4}\right) \sqrt{1-e_{p}^{2}}+ \\
+\frac{1}{4 e_{p}^{3}}\left(1+2 e_{p}^{2}\right) \text { arcsin } e_{p} \text { at } \rho<1, \\
\frac{1}{4 e_{p}^{2}}\left(1+8 e_{p}^{2}+4 e_{p}^{4}\right) \sqrt{1+e_{p}^{2}}- \\
-\frac{1}{4 e_{p}^{3}}\left(1-2 e_{p}^{2}\right) \times \\
\times \ln \left(e_{p}+\sqrt{1+e_{p}^{2}}\right) \text { at } \rho>1 .
\end{array}\right.
\end{aligned}
$$

Here, $e_{p}=\left|1-\rho^{2}\right|^{1 / 2}$ is the eccentricity, and $\rho=$ $=R_{\perp} / R_{\|}$the ratio of radii. Since the relation between $e_{p}$ and $\rho$ is ambiguous (two different $\rho$-values correspond to the same $e_{p}$-value), it is convenient to take the curvature radius ratio $\rho=R_{\perp} / R_{\|}$rather than the eccentricity $e_{p}$ as an independent parameter characterizing the influence of the nanoparticle shape on the absorption.

In the case of spherical nanoparticles, $R_{\perp}=R_{\|}=$ $=a$ (i.e. $e_{p} \rightarrow 0$ ). From Eq. (18), we obtain that $\chi_{H} \rightarrow 2 / 3$ and $\eta_{H} \rightarrow 8 / 3$. Using those parameter values and Eq. (18), we obtain the result of work [10]:

$W_{m}^{(0)}=\frac{3}{64} V a \frac{n e^{2} v_{\mathrm{F}}}{m c^{2}}\left(H^{(0)}\right)^{2}$.

Below, while studying the dependence of the nanoparticle absorption on the nanoparticle shape, it is more convenient to take the ratio between the energy absorbed by a spheroidal particle and the energy absorbed by a spherical particle with the same volume

ISSN 2071-0194. Ukr. J. Phys. 2018. Vol. 63, No. 10 
as the sought quantity. Hence, the matter concerns the ratio between expressions (18) and (21),

$$
\begin{aligned}
& \frac{W_{m}}{W_{m}^{(0)}}=\frac{3}{2} \rho^{1 / 3}\left[\chi_{H}\left(H_{\|}^{(0)}\right)^{2}+\right. \\
& \left.+\eta_{H}\left(1+\rho^{2}\right)^{-2}\left(H_{\perp}^{(0)}\right)^{2}\right] /\left(H^{(0)}\right)^{2} .
\end{aligned}
$$

When deriving this formula, we took into account that

$$
\frac{R_{\perp}}{a}=\frac{R_{\perp}}{\left(R_{\perp}^{2} R_{\|}\right)^{1 / 3}}=\rho^{1 / 3} .
$$

Ratio (22) also equals to the ratio between the absorption coefficients of the spheroidal and spherical nanoparticles. In the coordinate frame oriented along the principal spheroid axes,

$H_{\|}^{(0)}=H^{(0)} \cos \theta, \quad H_{\perp}^{(0)}=H^{(0)} \sin \theta$,

where $\theta$ is the angle between the vector $\mathbf{H}^{(0)}$ and the spheroid revolution axis.

Taking all the aforesaid into account, we obtain

$$
\frac{W_{m}}{W_{m}^{(0)}}=\frac{3}{2} \rho^{1 / 3}\left[\chi_{H} \cos ^{2} \theta+\frac{\eta_{H}}{\left(1+\rho^{2}\right)^{2}} \sin ^{2} \theta\right] .
$$

We also assume that spheroidal metal nanoparticles are oriented chaotically in the dielectric matrix. This assumption gives us ground to average expression (24) over all $\theta$-angle values. As a result, after averaging over the orientations of the spheroid symmetry axis, ratio (24) between the magnetic absorption by a spheroidal metal nanoparticle and the similar absorption by a spherical particle takes the form

$\left\langle\frac{W_{m}}{W_{m}^{(0)}}\right\rangle=\frac{3}{4} \rho^{1 / 3}\left[\chi_{H}+\frac{\eta_{H}}{\left(1+\rho^{2}\right)^{2}}\right]$.

It should be noted that the magnetic absorption (25) does not depend on the frequency in interval (17). This fact can be easily understand on the example of spherical nanoparticles. In expression (1), the magnetic absorption is described by the second term. In the frequency range (17), as one can see from Eq. (2), $\omega \epsilon^{\prime \prime} \sim \nu \omega_{p}^{2} / \omega^{2}$. Substituting this expression into Eq. (1), we obtain that the frequency dependence of the magnetic absorption disappears. Note also that formula (1) was derived in the case where

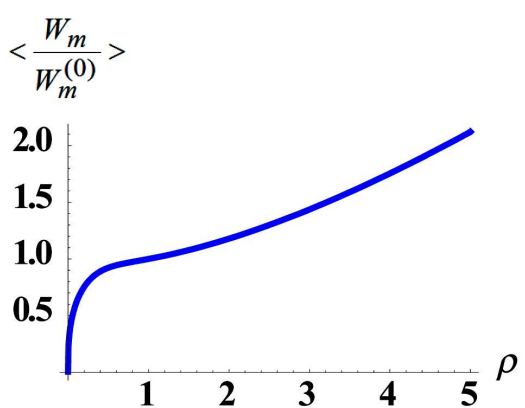

Fig. 1. Dependence of the averaged ratio $\left\langle W_{m} / W_{m}^{(0)}\right\rangle$ between the energies of magnetic absorption by spheroidal and spherical metal nanoparticles on the ratio between the curvature radii of the spheroid $\rho=R_{\perp} / R_{\|}$

the nanoparticle size is much larger than the electron free path. In the general case, i.e. at an arbitrary ratio between the free-electron path length and the nanoparticle size, the problem of light scattering by a spherical metal particle was solved in work [12].

\section{Results of Computational Experiment and Their Interpretation}

Figure 1 exhibits the dependence of the ratio $\left\langle K_{m}\right\rangle / K_{m}^{(0)}=\left\langle W_{m} / W_{m}^{(0)}\right\rangle$ on the spheroid curvature radius ratio $\rho=R_{\perp} / R_{\|}$. Here, $K_{m}^{(0)}$ is the coefficient of magnetic absorption by a spherical particle with the volume equal to the volume of the ellipsoid of revolution.

Before analyzing the plot in Fig. 1, we would like to make the following brief remark. As was indicated in Introduction, it is convenient to develop the theory for ellipsoidal particles, because the results obtained for this shape can be extended to a wide range of nanoparticle forms from discoid to rod-like ones by changing the curvature radii of the ellipsoid . Furthermore, we emphasized that the ratio of curvature radii $\rho=R_{\perp} / R$ is expedient to be taken as a parameter rather than the eccentricity $e_{p}$. Therefore, it is natural to say that, with the growth of $\rho$, the nanoparticle becomes more oblate. Hence, from Fig. 1, the following conclusion can be drawn. The more oblate the spheroidal metal nanoparticle, the larger is its averaged magnetic absorption. This growth has a smooth character at $\rho>0.5$.

Of much greater interest is the spatial distribution of the ratio between the energies absorbed by 


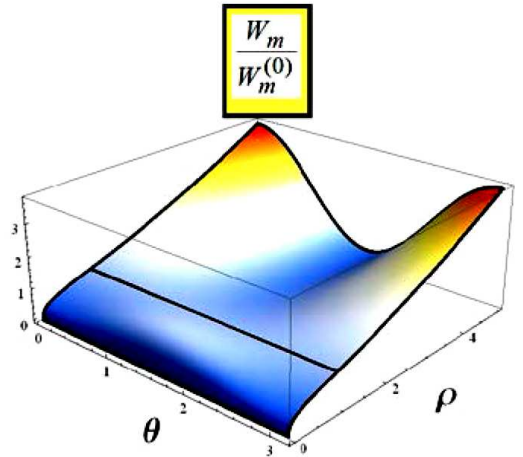

$a$

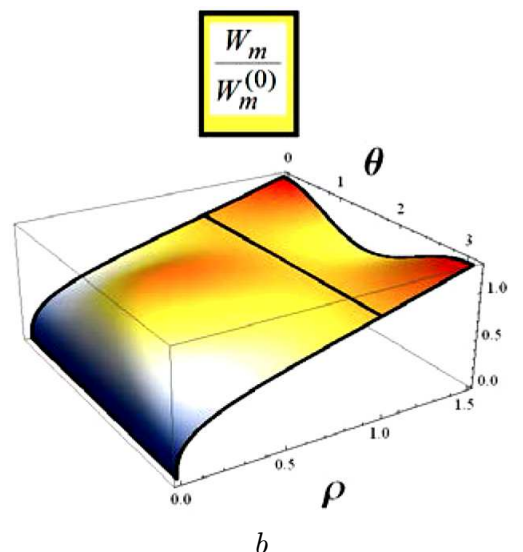

$b$

Fig. 2. Spatial distribution of the ratio between the energies of magnetic absorption by spheroidal and spherical metal nanoparticles, $W_{m}(\rho, \theta) / W_{m}^{(0)}$. Panel $b(0<\rho<1.5)$ is a scaled up section of panel $a(0<\rho<5)$

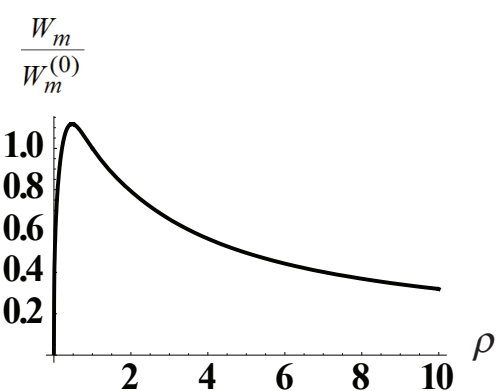

Fig. 3. Dependence of the ratio $W_{m}(\pi / 2, \rho) / W_{m}^{(0)}$ between the energies of magnetic absorption by spheroidal and spherical metal nanoparticles on the ratio between the curvature radii of the spheroid $\rho=R_{\perp} / R_{\|}$at $\theta=\pi / 2$

the spheroidal and spherical particles, $W_{m}(\rho, \theta) / W_{m}^{(0)}$ (Fig. 2). To what extent the geometric surface depicted in Fig. 2 is adequate to physical ideas can be judged on the basis of the following facts. First of

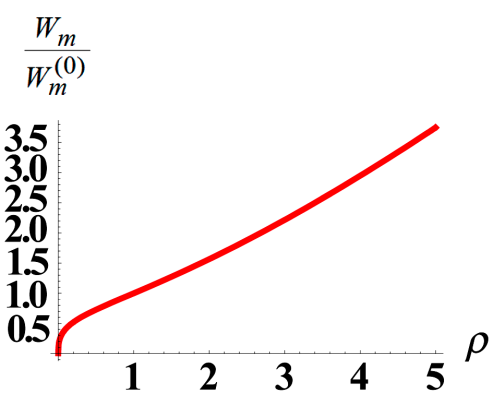

Fig. 4. Dependence of the ratio $W_{m}(\pi, \rho) / W_{m}^{(0)}$ between the energies of magnetic absorption by spheroidal and spherical metal nanoparticles on the ratio between the curvature radii of the spheroid $\rho=R_{\perp} / R_{\|}$at $\theta=0$ and $\pi$

all, the attention is attracted to a boundary between two parts of the surface: the convex, at $0 \leq \rho<1$, and concave, at $\rho>1$, regions. This boundary is a straight line, with every point of which satisfying the formula

$\frac{W_{m}(\rho=1, \theta)}{W_{m}^{(0)}}=1, \quad \theta \in[0, \pi]$.

From the physical point of view, this relation is confirmed by a simple fact: for a particle with $\rho=$ $=R_{\perp} / R_{\|}=1$, the absorbed energy equals the energy absorbed by a spherical particle independently of the angle $\theta$ between the vector $\mathbf{H}^{(0)}$ and the axis of sphere revolution: $W_{m}(\rho=1, \theta)=W_{m}^{(0)}$.

Another interesting fact consists in the growth of the energy absorbed by a spheroidal nanoparticle with the increasing $\rho$ (the nanoparticle becomes more oblate) at any values of the angle $\theta \in\{[0, \pi / 2) \cup$ $\cup(\pi / 2, \pi]\}$. The only value of $\theta$, at which the curve $\frac{W_{m}(\rho, \theta=\pi / 2)}{W_{m}^{(0)}}$ asymptotically approaches zero, is $\theta=$ $=\pi / 2^{m}$ (Fig. 3).

The maximum growth of the magnetic absorption by a spheroidal metal nanoparticle is observed at two values of the angle $\theta$ : 0 and $\pi$ (Fig. 4). Here, this growth is identical.

Of course, if a very oblate (almost flat) particle is oriented normally to the magnetic field vector $(\theta=$ $=0$ or $\pi$ ), then the effect of its interaction with this field is maximum. But if the same particle (almost flat) is oriented along the magnetic field vector $(\theta=$ $=\pi / 2)$, then the effect of its interaction with this field is small.

ISSN 2071-0194. Ukr. J. Phys. 2018. Vol. 63, No. 10 


\section{Conclusions}

- The work contains the results of theoretical studies concerning the dependence of the magnetic light absorption by a metal nanoparticle with regard for the influence of a nanoparticle shape on this process. In particular, the effect of spheroidal nanoparticles is analyzed. It is convenient to develop the theory for the ellipsoidal form, because the results obtained for this form can be extended to a wide range of nanoparticle forms (from discoid to rod-like ones), The curvature radii of the ellipsoid are varied in this case by means of only the ratio between them, $\rho=R_{\perp} / R_{\|}$, because the nanoparticle volume remains constant.

- An expression for the ratio between the energies absorbed by the spheroidal and spherical particles (with the same volumes) is derived, which is suitable for applications.

- An expression is obtained for the calculation of the averaged value of the ratio between the magnetic absorption by the spheroidal and spherical metal nanoparticles in terms of the ratio between the curvature radii of the spheroid $\rho=R_{\perp} / R_{\|}$.

- The work also contains the results of computational experiments. The most interesting result of the latter is the growth of the energy absorption by the spheroidal nanoparticle with the growth of $\rho$ (its disk-like character) at an arbitrary value of the angle $\theta \in\{[0, \pi / 2) \cup(\pi / 2, \pi]\}$. The only value, at which the curve $\frac{W_{m}(\rho, \theta=\pi / 2)}{W_{m}^{(0)}}$ asymptotically approaches zero, is $\theta=\pi / 2$.

To summarize, in our earlier work [9], we analyzed the dependence of the electric absorption of metal nanoparticles on their shape. In contrast to the magnetic absorption, the electric absorption of nanoparticles drastically depends on their shape. This fact takes place, because the electric absorption in the visible frequency range is mainly determined by plasma resonances. At the same time, the frequencies of plasma resonances, their half-widths, and their number depend on the nanoparticle shape. By changing the parameter $\rho$ (at a fixed frequency $\omega$ ), we can both enter into the resonance regime with characteristic plasma frequencies and exit from it. This fact results in a drastic dependence of the electric absorption on the metal nanoparticle shape.

On the other hand, the magnetic absorption in the frequency range (17), where it can be substantial, does not depend on the frequency at all. The dependence of the magnetic absorption on the nanoparticle shape mainly manifests itself in the angular zone, as one can see from Fig. 2, $a$.

1. C.F. Boren, D.R. Huffman. Absorption and Scattering of Light by Small Particles (Wiley, 1983).

2. H. C. van de Hulst. Light Scattering by Small Particles (Wiley, 1957).

3. V.V. Klimov. Nanoplasmonics (Nauka, 2012) (in Russian).

4. E.F. Venger, A.V. Goncharenko, M.L. Dmitruk. Optics of Small Particles and Disperse Media (Naukova Dumka, 1999) (in Ukrainian).

5. L.D. Landau, E.M. Lifshitz. Electrodynamics of Continuous Media (Pergamon Press, 1984).

6. G. Mie. Beiträge zur Optik trüber Medien, speziell kolloidaler Metallösungen. Ann. Phys. 25, 377 (1908).

7. P.M. Tomchuk, N.I. Grigorchuk. Shape and size effects on the energy absorption by small metallic particles. Phys. Rev. B 73, 155423 (2006).

8. P.M. Tomchuk, D.V. Butenko. Nonlocal effects in metallic nanoparticles: The kinetic approach outlook. Int. J. Mod. Phys. B 31, 1750029 (2017).

9. P.M. Tomchuk, V.N. Starkov. Influence of shape spread in an ensemble of metal nanoparticles on their optical properties. Ukr. Fiz. Zh. 63, 204 (2018) (in Ukrainian).

10. P.M. Tomchuk, B.P. Tomchuk. Optical absorption by small metal particles. Zh. Èksp. Teor. Fiz. 112, 661 (1997) (in Russian).

11. M.I. Grigorchuk, P.M. Tomchuk. Absorption and radiation emission by small ellipsoidal metal particles. J. Phys. Stud. 9, 135 (2005) (in Ukrainian).

12. I.A. Kuznetsova, M.E. Lebedev, A.A. Yushkanov. Influence of kinetic boundary conditions on the scattering crosssection of electromagnetic radiation at small metal particle. Zh. Tekhn. Fiz. 85, No. 9, 1 (2015) (in Russian).

Received 29.09.18.

Translated from Ukrainian by O.I. Voitenko

П.М. Томчук, В.М. Старков

МАГНІТНЕ ПОГЛИНАННЯ

МЕТАЛЕВИХ НАНОЧАСТИНОК

Р ез ю м е

Побудовано і теоретично досліджено залежність магнітного поглинання від відношення радіусів кривизни сфероїдальних металевих наночастинок, а також залежність від кута між віссю симетрії сфероїда і вектором магнітного поля електромагнітної хвилі. Цікавим результатом дослідження $\epsilon$ зростання поглинання енергії сфероїдальною наночастинкою у міру зростання її дископодібності. 